\title{
TWO-DIMENSIONAL RIEMANN PROBLEM FOR PRESSURELESS GAS DYNAMICS EQUATIONS WITH FUNCTIONAL SOLUTIONS
}

\author{
BY
}

JIAXIN HU

\begin{abstract}
Young Scientist Laboratory of Mathematical Sciences, Wuhan Institute of Physics and Mathematics, Academia Sinica, China
\end{abstract}

\begin{abstract}
The Riemann problem for a two-dimensional hyperbolic system of pressureless gas dynamics equations is considered here. Riemann solutions are constructed for any piecewise-constant initial data having two discontinuity rays with the origin as vertex. Non-classical waves (labelled as Dirac-shock waves) appear in some solutions. The entropy conditions for the Dirac-shock waves are given. Uniqueness of Riemann solutions containing Dirac-shock waves fails.
\end{abstract}

1. Introduction. We are concerned with a $3 \times 3$ two-dimensional hyperbolic system of the form

$$
\left\{\begin{array}{l}
\rho_{t}+(\rho u)_{x}+(\rho v)_{y}=0 \\
(\rho u)_{t}+\left(\rho u^{2}\right)_{x}+(\rho u v)_{y}=0 \\
(\rho v)_{t}+(\rho u v)_{x}+\left(\rho v^{2}\right)_{y}=0, \quad(x, y, t) \in R^{2} \times R_{+}
\end{array}\right.
$$

with initial data

$\left.(\rho, u, v)\right|_{t=0}=\left(\rho_{0}(x, y), u_{0}(x, y), v_{0}(x, y)\right)=\left\{\begin{array}{l}\left(\rho_{2}, u_{2}, v_{2}\right), x>0, y>0, \\ \left(\rho_{1}, u_{1}, v_{1}\right), \text { otherwise }, \rho_{1} \geq 0, \rho_{2} \geq 0 .\end{array}\right.$

We call (1.1), (1.2) a Riemann problem.

System (1.1) is a special form of the equations of fluid dynamics. We recall that the equations of fluid dynamics in Eulerian form with two space coordinates read

$$
\left\{\begin{array}{l}
\rho_{t}+(\rho u)_{x}+(\rho v)_{y}=0 \text { (conservation of mass) } \\
(\rho u)_{t}+\left(\rho u^{2}+p\right)_{x}+(\rho u v)_{y}=0 \\
(\rho v)_{t}+(\rho u v)_{x}+\left(\rho v^{2}+p\right)_{y}=0 \text { (conservation of momentum) }
\end{array}\right.
$$

Received December 30, 1997.

2000 Mathematics Subject Classification. Primary 35L65.

The author was supported by CNSF(Youth). 
where $\rho$ stands for the density and $(u, v)$ for the velocity of the fluid [1]. The density $\rho$ is nonnegative; the regions in the physical space where $\rho=0$ are identified with vacuum regions of the flow. As we know, a flow is formed by two kinds of effects: one is the effect of inertia and the other is the effect of pressure difference. If we neglect the effect of pressure difference in (1.4) (that is, the pressure $\rho$ is constant), (1.3) and (1.4) are reduced to (1.1). The system (1.1) also describes other important physical phenomena; see [9], [10] and the references therein.

For smooth solutions, (1.1) can be written as

$$
U_{t}+A U_{x}+B U_{y}=0
$$

where

$$
U=\left(\begin{array}{l}
\rho \\
u \\
v
\end{array}\right), \quad A=\left(\begin{array}{lll}
u & \rho & 0 \\
0 & u & 0 \\
0 & 0 & u
\end{array}\right), \quad B=\left(\begin{array}{lll}
v & 0 & \rho \\
0 & v & 0 \\
0 & 0 & v
\end{array}\right) .
$$

So the characteristic equation of $(1.1)$ in the direction $(\mu, \nu)$ is

$$
\begin{aligned}
|\lambda E-\mu A-\nu B| & =\left|\begin{array}{ccc}
\lambda-\mu u-\nu v & -\mu \rho & -\nu \rho \\
0 & \lambda-\mu u-\nu v & 0 \\
0 & 0 & \lambda-\mu u-\nu v
\end{array}\right| \\
& =(\lambda-\mu u-\nu v)^{3},
\end{aligned}
$$

which implies that the system (1.1) has a triple eigenvalue $\lambda=\mu u+\nu v$. The corresponding right eigenvector of $\lambda$ is $r=(a, \nu,-\mu)^{\mathrm{T}}, a$ being any real number, and so $\nabla \lambda \cdot r \equiv 0$. Thus, the system (1.1) is nonstrictly hyperbolic and linearly degenerate in the sense defined in [8].

In order to solve (1.1), (1.2), the corresponding one-dimensional Riemann problems have to be considered. In [4], the author constructed the solutions of the system

$$
\left\{\begin{array}{l}
\rho_{t}+(\rho u)_{x}=0, \\
(\rho u)_{t}+\left(\rho u^{2}\right)_{x}=0, \\
(\rho v)_{t}+(\rho u v)_{x}=0,(x, t) \in R \times R_{+},
\end{array}\right.
$$

with initial data

$$
\left.(\rho, u, v)\right|_{t=0}=\left\{\begin{array}{l}
\left(\rho_{2}, u_{2}, v_{2}\right), x>0, \\
\left(\rho_{1}, u_{1}, v_{1}\right), x \leq 0,
\end{array}\right.
$$

by employing the viscosity vanishing approach, and found that non-classical solutions appeared for some initial data. In other words, Riemann solutions no longer belong to locally bounded spaces $L_{\mathrm{loc}}^{\infty}\left(R \times R_{+}\right)$, but can be viewed as bounded functions plus weighted Dirac measures. The introduction of Dirac measures into weak solutions can be traced back as early as in 1977 in Korchinski's thesis [6]. But this new idea did not receive much attention. In recent years, people have justified this introduction in studying some one-dimensional hyperbolic systems of conservation laws by applying various tools such as the viscosity approach [5], [7], potentials and Lebesgue-Stieljtes integrals [2], [3].

In the present paper, we shall construct the solution to (1.1), (1.2). Like the onedimensional case [4], it is impossible to reach the uniqueness of Riemann solutions to 
(1.1), (1.2) when vacuum regions arise. The non-classical waves, which are labelled as the Dirac-shock waves, appear in some solutions. However, there is a striking feature for a Dirac-shock wave, on which there exist infinitely many expressions of the solution of (1.1), (1.2). This is considerably different from what is the case for a one-dimensional non-classical wave of (1.5), (1.6) on which the expression of the solution is unique [4].

By a weak solution to (1.1), (1.2), we mean piecewise smooth functions $(\rho(x, y, t)$, $u(x, y, t), v(x, y, t))$, perhaps unbounded on some planes in $(x, y, t)$-space, satisfying

$$
\begin{array}{r}
\int_{0}^{\infty} \iint_{R^{2}}\left(\rho \varphi_{t}+\rho u \varphi_{x}+\rho v \varphi_{y}\right) d x d y d t=0, \\
\int_{0}^{\infty} \iint_{R^{2}}\left(\rho u \varphi_{t}+\rho u^{2} \varphi_{x}+\rho u v \varphi_{y}\right) d x d y d t=0, \\
\int_{0}^{\infty} \iint_{R^{2}}\left(\rho v \varphi_{t}+\rho u v \varphi_{x}+\rho v^{2} \varphi_{y}\right) d x d y d t=0
\end{array}
$$

for any $\varphi(x, y, t) \in C_{0}^{\infty}\left(R^{2} \times R_{+}\right)$and the following initial condition:

every discontinuous plane of functions $(\rho(x, y, t), u(x, y, t), v(x, y, t))$ for small time $t>0$ intersects the plane $t=0$ at the origin or on the discontinuous line $x=0, y \geq 0$, or $y=0, x \geq 0$ of the initial data.

In the following context, we shall see that only $\rho(x, y, t)$ is unbounded on some planes (called Dirac-shock waves) while $u(x, y, t)$ and $v(x, y, t)$ are always bounded on $R^{2} \times R_{+}$. Also, $\rho(x, y, t)$ can be viewed as a bounded function in $L^{\infty}\left(R^{2} \times R_{+}\right)$plus a bounded linear functional on $C_{0}^{\infty}\left(R^{2} \times R_{+}\right)$with the Dirac-shock waves as its supports. At this time, there arises a concurrent problem of how to define the product of a linear bounded functional on $C_{0}^{\infty}\left(R^{2} \times R_{+}\right)$by a discontinuous function. To overcome this problem, we give

Definition 1. Let $\tau$ be a bounded linear functional on $C_{0}^{\infty}\left(R^{2} \times R_{+}\right)$with $K \subset$ $R^{2} \times R_{+}$as its support and $w(x, y, t)$ a discontinuous function in $R^{2} \times R_{+}$but smooth on $K$. Then we define the product $w \tau$ by

$$
\langle w \tau, \varphi\rangle=\left\langle\tau,\left.(w \varphi)\right|_{K}\right\rangle
$$

for any $\varphi(x, y, t) \in C_{0}^{\infty}\left(R^{2} \times R_{+}\right)$. We note that the right-hand side of (1.9) makes sense since $(w \varphi)(x, y, t)$ is smooth on $K$. Thus $w \tau$ is well defined.

This paper is organized as follows: Sec. 2 gives some preliminaries while Sec. 3 constructs the solution to (1.1), (1.2) according to the classification of the initial data. It is easily seen that if two states of the initial data are vacuum states, i.e., $\rho_{1}=\rho_{2}=0$, then $(\rho, u, v)=(0, u(x, y, t), v(x, y, t))$ is a solution of $(1.1),(1.2)$. Here $u(x, y, t)$ and $v(x, y, t)$ are arbitrary. In the following discussion, we assume that only one state of the initial data is the vacuum state.

\section{Preliminaries.}

2.1. Self-similar solutions. Since (1.1) and (1.2) are invariant under the transformation $x \rightarrow \alpha x^{\prime}, y \rightarrow \alpha y^{\prime}, t \rightarrow \alpha t^{\prime}, \alpha>0$, we should seek self-similar solutions. Upon setting $\xi=x / t, \eta=y / t$, the system (1.1) becomes

$$
A U_{\xi}+B U_{\eta}=0
$$


where

$$
\begin{gathered}
U=\left(\begin{array}{l}
\rho \\
u \\
v
\end{array}\right), \quad A=\left(\begin{array}{ccc}
u-\xi & \rho & 0 \\
0 & \rho(u-\xi) & 0 \\
0 & 0 & \rho(u-\xi)
\end{array}\right), \\
B=\left(\begin{array}{ccc}
v-\eta & 0 & \rho \\
0 & \rho(v-\eta) & 0 \\
0 & 0 & \rho(v-\eta)
\end{array}\right)
\end{gathered}
$$

and (1.2) changes to

$$
(\rho, u, v)(\xi, \eta) \rightarrow\left\{\begin{array}{c}
\left(\rho_{2}, u_{2}, v_{2}\right), \xi>0, \eta>0 \text { and } \xi^{2}+\eta^{2} \rightarrow \infty \\
\left(\rho_{1}, u_{1}, v_{1}\right), \xi<0 \text { and } \xi^{2}+\eta^{2} \rightarrow \infty, \text { or } \xi>0, \eta<0 \\
\text { and } \xi^{2}+\eta^{2} \rightarrow \infty
\end{array}\right.
$$

For self-similar solutions, the characteristic equation of $(2.1)$ is

$$
\begin{aligned}
|B-\lambda A| & =\left|\begin{array}{ccc}
v-\eta-\lambda(u-\xi) & -\lambda \rho & \rho \\
0 & \rho(v-\eta-\lambda(u-\xi)) & 0 \\
0 & 0 & \rho(v-\eta-\lambda(u-\xi))
\end{array}\right| \\
& =\rho^{2}(v-\eta-\lambda(u-\xi))^{3}
\end{aligned}
$$

and so (2.1) has a triple characteristic value $\lambda=(v-\eta) /(u-\xi)$. The corresponding right characteristic rector is $r=(a, 1, \lambda)^{\mathrm{T}}, a$ being any real number, and thus $\nabla \lambda \cdot r \equiv 0$. The characteristic curve, denoted by $\Gamma$, is defined by

$$
\frac{d \eta}{d \xi}=\lambda=\frac{v(\xi, \eta)-\eta}{u(\xi, \eta)-\xi}
$$

At the point $(\xi, \eta)$, we orient the direction of $\Gamma$ to point to $(u, v)$.

2.2. Classical waves. Let $(\rho, u, v)(x, y, t) \in L_{\mathrm{loc}}^{\infty}\left(R^{2} \times R_{+}\right)$be a solution of $(1.1),(1.2)$ and $\eta=\eta(\xi)$ (or equivalently $y=t \eta(x / t)$ ) a discontinuity surface of $(\rho, u, v)(x, y, t)$. Then the Rankine-Hugoniot condition is

$$
\begin{aligned}
(-\eta+\xi \sigma)[\rho]-\sigma[\rho u]+[\rho v] & =0 \\
(-\eta+\xi \sigma)[\rho u]-\sigma\left[\rho u^{2}\right]+[\rho u v] & =0 \\
(-\eta+\xi \sigma)[\rho v]-\sigma[\rho u v]+\left[\rho v^{2}\right] & =0
\end{aligned}
$$

or

$$
\left.\begin{array}{ccc}
v_{1}-\eta+\sigma\left(\xi-u_{1}\right) & \dot{\rho} & \rho \\
0 & \rho_{1}\left(v_{1}-\eta+\sigma\left(\xi-u_{1}\right)\right) & 0 \\
0 & 0 & \rho_{1}\left(v_{1}-\eta+\sigma\left(\xi-u_{1}\right)\right)
\end{array}\right)
$$


where $\sigma=\eta^{\prime}(\xi)$ and $[\rho]=\rho-\rho_{1}$ is the jump of $\rho$ across the discontinuity. By (2.4), it follows that

$$
\rho_{1}=0, \rho \neq 0, \sigma=\frac{v-\eta}{u-\xi} \quad\left(\text { or } \rho=0, \rho_{1} \neq 0, \sigma=\frac{v_{1}-\eta}{u_{1}-\xi}\right),
$$

or

$$
\rho_{1} \rho_{2}>0, \quad \sigma=\frac{v_{1}-\eta}{u_{1}-\xi}=\frac{v_{1}-v}{u_{1}-u} .
$$

The discontinuity given by (2.5) or (2.6) is called a contact discontinuity since the wave speed $\sigma$ coincides with the characteristic value $\lambda$ on either side of the discontinuity.

2.3. Dirac-shock waves. Given a point $P$ in the $(\xi, \eta)$-plane, sometimes two states cannot be connected by one or more than one contact discontinuity passing through $P$. One kind of new idea was proposed that the Riemann solution should take on some nonstandard values on the discontinuity line (classical wave) in the $(\xi, \eta)$-plane in order to satisfy the system in the sense of distributions (cf. [7], [6], [5]). Therefore, we can say that a non-classical wave shares the same equation with a classical one. Moreover, by the initial condition (2.2), a two-dimensional non-classical wave should agree with a one-dimensional non-classical wave at infinity. We notice that the entropy condition is that $u_{2}>u_{1}$ for the non-classical shock wave of the one-dimensional Riemann solution to (1.5), (1.6), and the non-classical discontinuity line is computed to be $\tau=\frac{\sqrt{\rho_{1}} u_{1}+\sqrt{\rho_{2}} u_{2}}{\sqrt{\rho_{1}}+\sqrt{\rho_{2}}}$ which satisfies the Lax entropy inequalities $u_{2}<\tau<u_{1}$ (see [4]). Based on these observations, we give

Definition 2. The straight line $\xi=\tau, a<\eta<b$ (or $\eta=\sigma, a<\xi<b$ ) in the $(\xi, \eta)$-plane, $a, b$ being real numbers (perhaps $a=-\infty, b=\infty$ ), is called a Dirac-shock wave, denoted by $\delta$, if such an entropy condition holds such that

$$
u^{-}>u^{+} \quad\left(\text { or } v^{-}>v^{+}\right)
$$

where $\left(\rho^{-}, u^{-}, v^{-}\right)$is the left (or lower) state connected with the right (or upper) state $\left(\rho^{+}, u^{+}, v^{+}\right)$by the wave $\delta$, and

$$
\tau=\frac{\sqrt{\rho^{+}} u^{+}+\sqrt{\rho^{-}} u^{-}}{\sqrt{\rho^{+}}+\sqrt{\rho^{-}}} \quad\left(\text { or } \sigma=\frac{\sqrt{\rho^{+}} v^{+}+\sqrt{\rho^{-}} v^{-}}{\sqrt{\rho^{+}}+\sqrt{\rho^{-}}}\right) .
$$

The entropy condition also can be described as the following: at any point of the Diracshock wave $\delta$, all characteristic lines come into this point. See Figure 2.1.

Here and below $\oplus$ stands for the state $\left(\rho^{+}, u^{+}, v^{+}\right)$and $(+)$for the point $(\xi, \eta)=$ $\left(u^{+}, v^{+}\right)$.

2.4. Classification of the initial data. According to the initial condition (2.2), twodimensional discontinuities coming from infinity in the $(\xi, \eta)$-plane are parallel to the $\xi$-axis or $\eta$-axis. In other words, at infinity, a two-dimensional wave shares the same equation with the corresponding one-dimensional one. In [4], we have constructed the solution of (1.5), (1.6) by the viscosity vanishing approach. The main results are summarized as follows. 
(i) $\rho_{1} \rho_{2}>0, u_{1}=u_{2}$. The solution to (1.5), (1.6) is depicted in Fig. 2.2.

(ii) $\rho_{1} \rho_{2}>0, u_{1}<u_{2}$. The solution to (1.5), (1.6) is depicted in Fig. 2.3. The vacuum region appears where $u(x, t)$ and $v(x, t)$ are arbitrary.
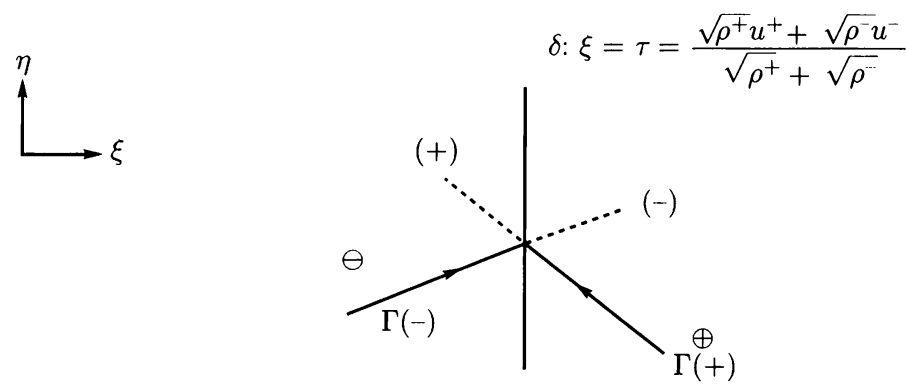

FIG. 2.1
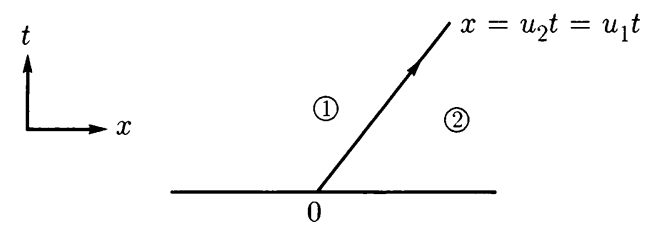

FIG. 2.2
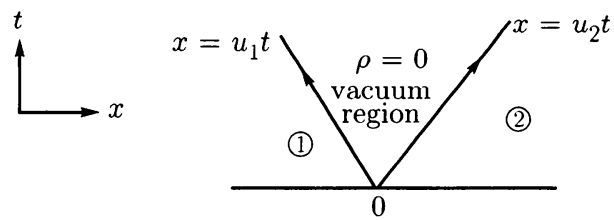

FIG. 2.3

(iii) $\rho_{1} \rho_{2}>0, u_{1}>u_{2}$. The solution to (1.5), (1.6) is depicted in Fig. 2.4.

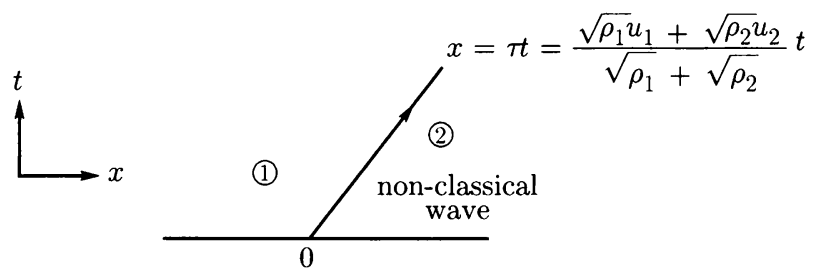

FIG. 2.4 
(iv) $\rho_{1}=0<\rho_{2}$. The solution to (1.5), (1.6) is depicted in Fig. 2.5 for $u_{1} \geq u_{2}$ and in Fig. 2.6 for $u_{1}<u_{2}$.
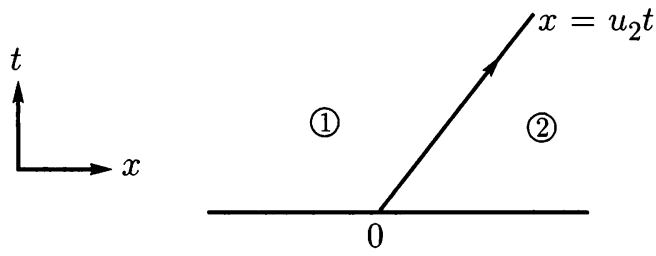

FIG. 2.5
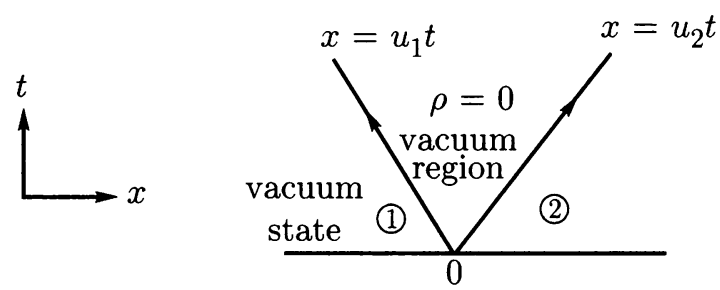

FIG. 2.6

(v) $\rho_{1}>0=\rho$. The solution to (1.5), (1.6) is depicted in Fig. 2.7 for $u_{1} \geq u_{2}$ and in Fig 2.8 for $u_{1}<u_{2}$.
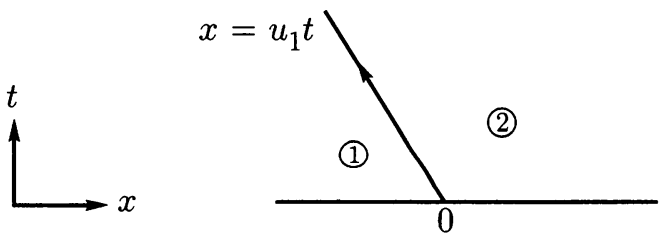

FIG. 2.7
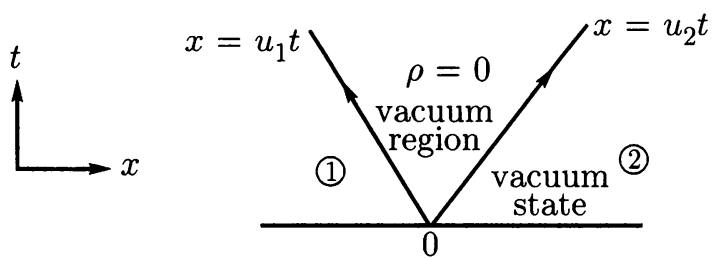

FIG. 2.8 
From above, we distinguish the initial data (1.2) as follows:
(1) $\rho_{1} \rho_{2}>0, u_{1}=u_{2}, v_{1}=v_{2}$,
(2) $\rho_{1} \rho_{2}>0, u_{1}=u_{2}, v_{1}<v_{2}$,
(3) $\rho_{1} \rho_{2}>0, u_{1}=u_{2}, v_{1}>v_{2}$,
(4) $\rho_{1} \rho_{2}>0, u_{1}<u_{2}, v_{1}<v_{2}$
(5) $\rho_{1} \rho_{2}>0, u_{1}<u_{2}, v_{1}>v_{2}$,
(6) $\rho_{1} \rho_{2}>0, u_{1}>u_{2}, v_{1}>v_{2}$,
(7) $\rho_{1}=0<\rho_{2}$
(8) $\rho_{1}>0=\rho_{2}$.

Other cases can be reduced to the above ones under the suitable transformations.

3. Riemann solutions to (1.1), (1.2). In this section we shall construct Riemann solutions to (1.1), (1.2) according to the classification of the initial data in Subsection 2.4 .

3.1. The case when $\rho_{1} \rho_{2}>0, u_{1}=u_{2}$ and $v_{1}=v_{2}$. The Riemann solution to (1.1), (1.2) is constructed as follows.

(i) There exist two contact discontinuities coming from infinity. One is parallel to the $\eta$-axis, denoted by $J_{12}$, and the other is parallel to the $\xi$-axis, denoted by $J_{21}$.

(ii) $J_{12}$ and $J_{21}$ vanish at the point $\left(u_{1}, v_{1}\right)=\left(v_{2}, v_{2}\right)$. See Fig. 3.1.
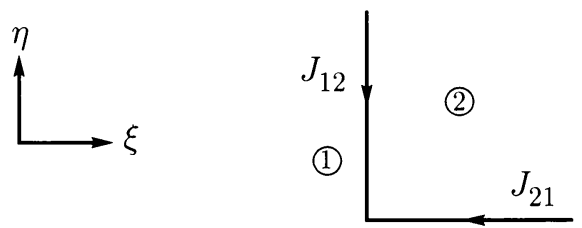

(1)

FIG. 3.1

3.2. The case when $\rho_{1} \rho_{2}>0, u_{1}=u_{2}$ and $v_{1}<v_{2}$. The Riemann solution to (1.1), (1.2) is constructed as follows.

(i) There exist two contact discontinuities (denoted by $J_{02}$ and $J_{10}$ respectively) coming from infinity, both of which are parallel to the $\xi$-axis, since $v_{1}<v_{2}$. At infinity, there is one contact discontinuity parallel to the $\eta$-axis, denoted by $J_{12}$.

(ii) $J_{12}$ and $J_{02}$ keep straight until they meet at the point $\left(u_{2}, v_{2}\right)$, and then a contact discontinuity, denoted by $J$, is produced. $J$ and $J_{10}$ vanish at the point $\left(u_{1}, v_{1}\right)$. See Fig. 3.2 .

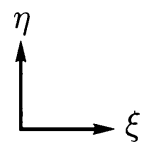

(1)

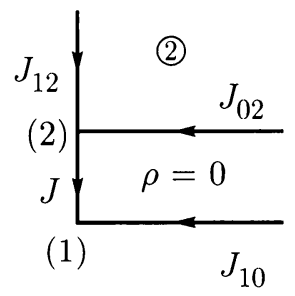


3.3. The case $\rho_{1} \rho_{2}>0, u_{1}=u_{2}$ and $v_{1}>v_{2}$. The Riemann solution to (1.1), (1.2) is easily constructed. See Fig. 3.3. Here $\sigma=\frac{\sqrt{\rho_{2}} v_{2}+\sqrt{\rho_{1}} v_{1}}{\sqrt{\rho_{2}}+\sqrt{\rho_{1}}}$.

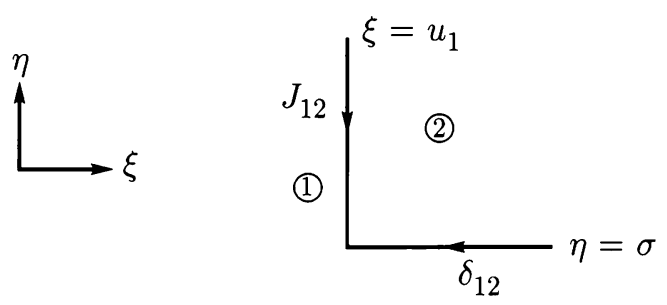

FIG. 3.3

Now we give the expression of the Riemann solution on the Dirac-shock wave $\delta_{12}$. To do this, we define

$$
\langle\delta, \varphi\rangle=\int_{0}^{\infty} d t \int_{u_{1} t}^{\infty} p(x, t) \varphi(x, \sigma t, t) d x
$$

for any $\varphi(x, y, t) \in C_{0}^{\infty}\left(R^{2} \times(0, \infty)\right)$, where $p(x, t)$ is a smooth function in $R \times R_{+}$ yet to be determined below. It is easily seen that $\delta$ is a bounded linear functional on $C_{0}^{\infty}\left(R^{2} \times R_{+}\right)$supported on the plane $K=\left\{(x, y, t): u_{1} t \leq x<\infty, y=\sigma t, 0 \leq t<\infty\right\}$. We set

$$
\begin{aligned}
(u(x, y, t), v(x, y, t))=\left\{\begin{array}{l}
\left(u_{1}, v_{2}\right), x>u_{1} t, y>\sigma t, 0<t<\infty \\
\left(u_{1}, \sigma\right), u_{1} t \leq x<\infty, y=\sigma t, 0<t<\infty \\
\left(u_{1}, v_{1}\right), \text { otherwise }
\end{array}\right. \\
\rho(x, y, t)=\bar{\rho}(x, y, t)+\lambda \delta
\end{aligned}
$$

where $\lambda$ is a constant (called the strength of $\delta$ ) to be determined below and $\bar{\rho}(x, y, t)$ is piecewise constant in $R^{2} \times R_{+}$:

$$
\bar{\rho}(x, y, t)=\left\{\begin{array}{l}
\rho_{2}, x>u_{1} t, y>\sigma t, 0<t<\infty \\
\rho_{1}, \text { otherwise. }
\end{array}\right.
$$

Let $f(x, y, z)$ be a smooth function in $R^{3}$. We set $g(x, y, z)=y f(x, y, z), h(x, y, z)=$ $z f(x, y, z)$, and $f_{1}=f\left(\rho_{1}, u_{1}, v_{1}\right), f_{2}=f\left(\rho_{2}, u_{2}, v_{2}\right)$, etc. We first compute the following integral which will be used later.

From (3.2), (3.4),

$$
\begin{aligned}
\int_{0}^{\infty} d t & \iint_{R^{2}}\left(f(\bar{\rho}, u, v) \varphi_{t}+g(\bar{\rho}, u, v) \varphi_{x}+h(\bar{\rho}, u, v) \varphi_{y}\right) d x d y \\
= & \int_{0}^{\infty} d t \int_{-\infty}^{u_{1} t} d x \int_{-\infty}^{\infty}\left(f_{1} \varphi_{t}+g_{1} \varphi_{x}+h_{1} \varphi_{y}\right) d y+\int_{0}^{\infty} d t \int_{u_{1} t}^{\infty} d x \int_{-\infty}^{\sigma t}\left(f_{1} \varphi_{t}+g_{1} \varphi_{x}\right. \\
& \left.+h_{1} \varphi_{y}\right) d y+\int_{0}^{\infty} d t \int_{u_{1} t}^{\infty} d x \int_{\sigma t}^{\infty}\left(f_{2} \varphi_{t}+g_{2} \varphi_{x}+h_{2} \varphi_{y}\right) d y \\
= & \int_{0}^{\infty} d t \int_{u_{1} t}^{\infty}\left(\sigma\left(f_{2}-f_{1}\right)+\left(h_{1}-h_{2}\right)\right) \varphi(x, \sigma t, t) d x
\end{aligned}
$$


since $g_{i}=u_{1} f_{i}, i=1,2$.

By (3.1), we have that for any $\varphi(x, y, t) \in C_{0}^{\infty}\left(R^{2} \times(0, \infty)\right)$,

$$
\begin{aligned}
\left\langle\delta, \varphi_{x}\right\rangle & =\int_{0}^{\infty} d t \int_{u_{1} t}^{\infty} p(x, t) \varphi_{x}(x, \sigma t, t) d x \\
& =-\int_{0}^{\infty} p\left(u_{1} t, t\right) \varphi\left(u_{1} t, \sigma t, t\right) d t-\int_{0}^{\infty} d t \int_{u_{1} t}^{\infty} \frac{\partial p}{\partial x}(x, t) \varphi(x, \sigma t, t) d x
\end{aligned}
$$

and

$$
\begin{aligned}
\left\langle\delta, \varphi_{t}+\sigma \varphi_{y}\right\rangle & =\int_{0}^{\infty} d t \int_{u_{1} t}^{\infty} p(x, t)\left(\varphi_{t}(x, \sigma t, t)+\sigma \varphi_{y}(x, \sigma t, t)\right) d x \\
& =\int_{0}^{\infty} d t \int_{u_{1} t}^{\infty} p(x, t) \frac{\partial}{\partial t} \varphi(x, \sigma t, t) d x \\
& =u_{1} \int_{0}^{\infty} \rho\left(u_{1} t, t\right) \varphi\left(u_{1} t, \sigma t, t\right) d t-\int_{0}^{\infty} d t \int_{u_{1} t}^{\infty} \frac{\partial p}{\partial t}(x, t) \varphi(x, \sigma t, t) d x
\end{aligned}
$$

Therefore, (3.6), (3.7) give

$$
\left\langle\delta, \varphi_{t}+u_{1} \varphi_{x}+\sigma \varphi_{y}\right\rangle=-\int_{0}^{\infty} d t \int_{u_{1} t}^{\infty}\left(\frac{\partial p}{\partial t}(x, t)+u_{1} \frac{\partial p}{\partial t}(x, t)\right) \varphi(x, \sigma t, t) d x
$$

Combining (3.2)-(3.4), using Definition 1, we obtain

$$
\begin{aligned}
\int_{0}^{\infty} & d t \iint_{R^{2}}\left(\rho \varphi_{t}+\rho u \varphi_{x}+\rho v \varphi_{y}\right) d x d y \\
& =\int_{0}^{\infty} d t \iint_{R^{2}}\left(\bar{\rho} \varphi_{t}+\bar{\rho} u \varphi_{x}+\bar{\rho} v \varphi_{y}\right) d x d y+\left\langle\lambda \delta, \varphi_{t}+u_{1} \varphi_{x}+\sigma \varphi_{y}\right\rangle .
\end{aligned}
$$

Taking $f(\rho, u, v)=\rho$ in (3.5), we get

$$
\begin{aligned}
\int_{0}^{\infty} & d t \iint_{R^{2}}\left(\bar{\rho} \varphi_{t}+\bar{\rho} u \varphi_{x}+\bar{\rho} v \varphi_{y}\right) d x d y \\
& =\sqrt{\rho_{1} \rho_{2}}\left(v_{1}-v_{2}\right) \int_{0}^{\infty} d t \int_{u_{1} t}^{\infty} \varphi(x, \sigma t, t) d x .
\end{aligned}
$$

Hence, we deduce from (3.8)-(3.10) that

$$
\begin{aligned}
\int_{0}^{\infty} & d t \iint_{R^{2}}\left(\rho \varphi_{t}+\rho u \varphi_{x}+\rho v \varphi_{y}\right) d x d y \\
= & \sqrt{\rho_{1} \rho_{2}}\left(v_{1}-v_{2}\right) \int_{0}^{\infty} d t \int_{u_{1} t}^{\infty} \varphi(x, \sigma t, t) d x \\
& -\lambda \int_{0}^{\infty} d t \int_{u_{1} t}^{\infty}\left(\frac{\partial p}{\partial t}+u_{1} \frac{\partial p}{\partial x}(x, t)\right) \varphi(x, \sigma t, t) d x \\
= & 0
\end{aligned}
$$


if we set $\lambda=\sqrt{\rho_{1} \rho_{2}}\left(v_{1}-v_{2}\right)$ and

$$
\frac{\partial p}{\partial t}(x, t)+u_{1} \frac{\partial p}{\partial x}=1
$$

Solving (3.11), it follows that

$$
p(x, t)=t+a\left(x-u_{1} t\right),
$$

where $a(x)$ is any smooth function in $R$. Therefore, (1.7a) holds for the $\lambda$ and $p(x, t)$ chosen above. Similarly, one can verify that (1.7b) and (1.7c) also hold for the above $\lambda$ and $p(x, t)$. Finally, (1.8) is trivial. Thus, $(\rho(x, y, t), u(x, y, t), v(x, y, t))$ given by (3.2), (3.3) are Riemann solutions to (1.1), (1.2).

3.4. The case when $\rho_{1} \rho_{2}>0, u_{1}<u_{2}$ and $v_{1}<v_{2}$. The Riemann solution to (1.1), (1.2) is depicted in Fig. 3.4.
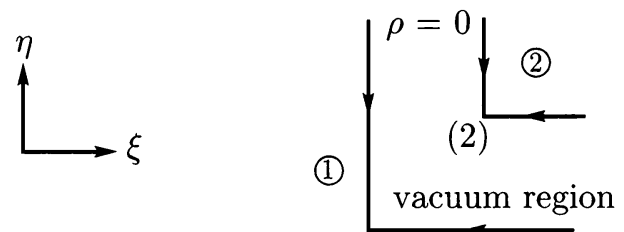

(1)

FIG. 3.4

3.5. The case when $\rho_{1} \rho_{2}>0, u_{1}<u_{2}$ and $v_{1}>v_{2}$. The Riemann solution to (1.1), (1.2) is constructed as follows:

(i) at infinity, there exist two contact discontinuities parallel to the $\eta$-axis, which are denoted by $J_{10}$ and $J_{02}$, respectively. At the same time, there is a Dirac-shock wave, denoted by $\delta_{12}$, that comes from infinity and is parallel to the $\xi$-axis.

(ii) $\delta_{12}$ and $J_{02}$ meet at the point $\left(u_{2}, \sigma\right), \sigma=\left(\sqrt{\rho_{2}} v_{2}+\sqrt{\rho_{1}} v_{1}\right) /\left(\sqrt{\rho_{2}}+\sqrt{\rho_{1}}\right)$, and then a contact discontinuity, denoted by $J$, is produced.

(iii) $J$ and $J_{10}$ vanish at the point $\left(u_{1}, v_{1}\right)$. See Fig. 3.5 .

3.6. The case when $\rho_{1} \rho_{2}>0, u_{1}>u_{2}$ and $v_{1}>v_{2}$. The Riemann solution to (1.1), (1.2) is easily constructed. See Fig. 3.6. Here $\tau=\left(\sqrt{\rho_{1}} u_{1}+\sqrt{\rho_{2}} u_{2}\right) /\left(\sqrt{\rho_{1}}+\sqrt{\rho_{2}}\right)$ and $\sigma=\left(\sqrt{\rho_{1}} v_{1}+\sqrt{\rho_{2}} v_{2}\right) /\left(\sqrt{\rho_{1}}+\sqrt{\rho_{2}}\right)$.
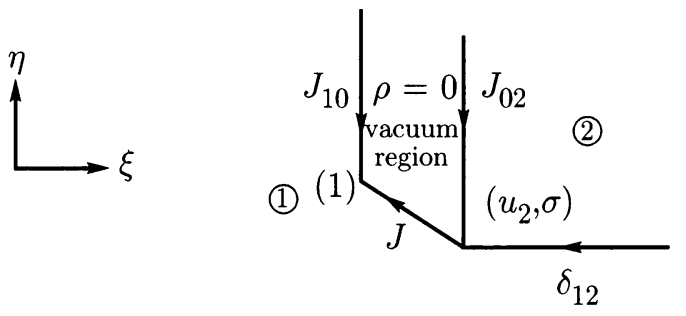

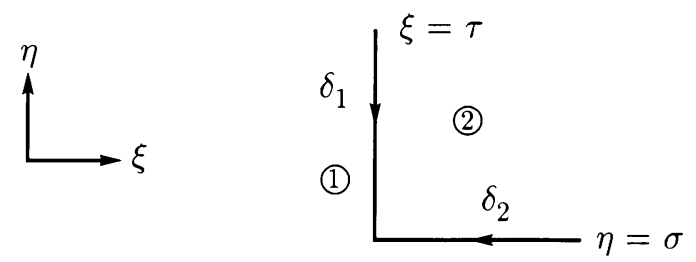

FIG. 3.6

Similar to the case in Subsection 3.3, the Riemann solution $(\rho(x, y, t), u(x, y, t)$, $v(x, y, t))$ is given by

$$
\begin{gathered}
(u(x, y, t), v(x, y, t))= \begin{cases}\left(u_{2}, v_{2}\right), & x>\tau t, y>\sigma t, 0<t<\infty, \\
(\tau, \sigma), & x=\tau t, \sigma t \leq y<\infty, 0<t<\infty, \\
& \tau t \leq x<\infty, y=\sigma t, 0<t<\infty, \\
\left(u_{1}, v_{1}\right), & \text { otherwise, }\end{cases} \\
\rho(x, y, t)=\bar{\rho}(x, y, t)+\lambda_{1} \delta_{1}+\lambda_{2} \delta_{2},
\end{gathered}
$$

where $\lambda_{1}=\sqrt{\rho_{1} \rho_{2}}\left(u_{1}-u_{2}\right), \lambda_{2}=\sqrt{\rho_{1} \rho_{2}}\left(v_{1}-v_{2}\right)$ and $\delta_{1}, \delta_{2}$ are determined by

$$
\begin{gathered}
\left\langle\delta_{1}, \varphi\right\rangle=\int_{0}^{\infty} d t \int_{\sigma t}^{\infty}(t+a(y-\sigma t)) \varphi(\tau t, y, t) d y \\
\left\langle\delta_{2}, \varphi\right\rangle=\int_{0}^{\infty} d t \int_{\tau t}^{\infty}(t+b(x-\tau t)) \varphi(x, \sigma t, t) d x, \quad \varphi(x, y, t) \in C_{0}^{\infty}\left(R^{2} \times R_{+}\right) .
\end{gathered}
$$

Here $a(x)$ and $b(x)$ are any smooth functions in $R$. So it is impossible to expect the uniqueness of Riemann solutions to (1.1), (1.2) since $\rho(x, y, t)$ is not determined uniquely.

3.7. The case when $\rho_{1}=0<\rho_{2}$. The Riemann solution to (1.1), (1.2) can be constructed by perturbing the initial data. Let $\left(\rho_{\delta}(x, y, t), u_{\delta}(x, y, t), v_{\delta}(x, y, t)\right)$ be a solution to (1.1) with the initial data

$$
\left.\left(\rho_{\delta}, u_{\delta}, v_{\delta}\right)\right|_{t=0}=\left\{\begin{array}{l}
\left(\rho_{2}, u_{2}, v_{2}\right), \quad x>0, y>0 \\
\left(\delta, u_{1}, v_{1}\right), \quad \text { otherwise }, \delta>0
\end{array}\right.
$$

We denote the weak-star limit in $\left(C_{0}^{\infty}\left(R^{2} \times R_{+}\right)\right)^{\prime}$ of $\left(\rho_{\delta}(x, y, t), u_{\delta}(x, y, t), v_{\delta}(x, y, t)\right)$ by $(\rho(x, y, t), u(x, y, t), v(x, y, t))$. It is easily seen that $(\rho(x, y, t), u(x, y, t), v(x, y, t))$ is the solution to (1.1), (1.2) with $\rho_{1}=0$. From the structure of $\left(\rho_{\delta}(x, y, t), u_{\delta}(x, y, t)\right.$, $\left.v_{\delta}(x, y, t)\right)$ obtained in Subsections 3.1-3.6, we get 
(a) $u_{1} \geq u_{2}, v_{1} \geq v_{2}$. The solution to (1.1), (1.2) is depicted in Fig. 3.7(a).
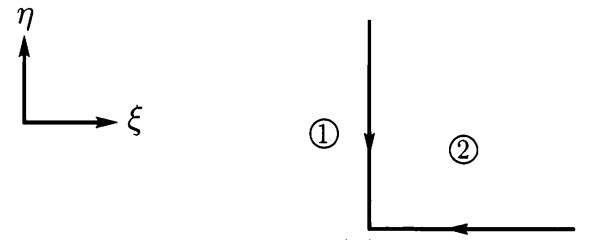

(2)

FIG. 3.7(a)

(b) $u_{1} \geq u_{2}, v_{1}<v_{2}$. The solution (1.1), (1.2) is depicted in Fig. 3.7(b).
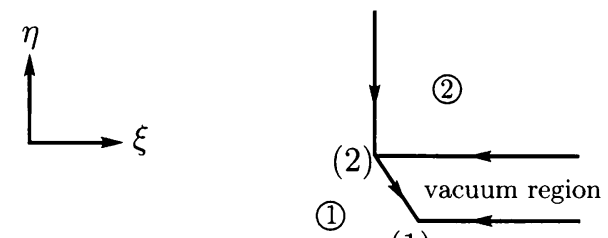

(1)

FIG. 3.7(b)

(c) $u_{1}<u_{2}, v_{1} \geq v_{2}$. The solution (1.1), (1.2) is depicted in Fig. 3.7(c).
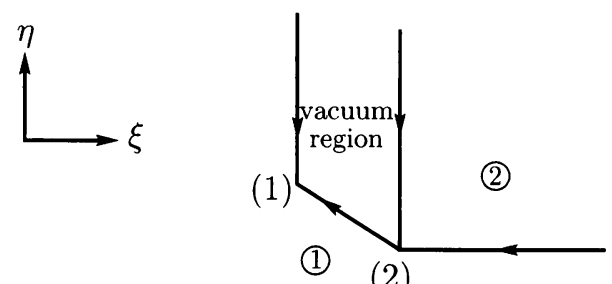

(2)

FIG. $3.7(\mathrm{c})$

(d) $u_{1}<u_{2}, v_{1}<v_{2}$. The solution (1.1), (1.2) is depicted in Fig. 3.7(d).
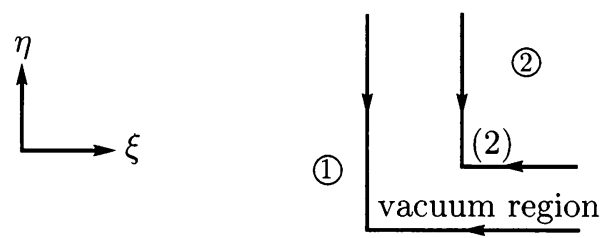

(1)

FIG. 3.7(d)

The Riemann solution to $(1.1),(1.2)$ for the case $\rho_{1}>0=\rho_{2}$ can be constructed similarly. We omit the details. 
Acknowledgment. I am grateful to Professor Xiaqi Ding for his persistent encouragement. Also, I would like to thank the anonymous referee for his (or her) valuable comments and suggestions.

\section{REFERENCES}

[1] R. Courant and K. O. Friedrichs, Supersonic flow and shock waves, Applied Mathematical Sciences, Vol. 21, Springer-Verlag, New York, 1976

[2] X. Q. Ding, On a non-strictly hyperbolic system, preprint, Dept. of Math., University of Jyvaskyla, Finland, No. 167, 1993

[3] X. Q. Ding and Z. Wang, Existence and uniqueness of discontinuous solutions defined by LebesgueStieltjes integral, Sci. China Ser. A 39, 807-819 (1996)

[4] J. X. Hu, One-dimensional Riemann problem for the equations of constant pressure fluid dynamics with functional solutions by the viscosity method, Acta Applicandae Mathematicae 55, 209-229 (1999)

[5] J. X. Hu, A limiting viscosity approach to Riemann solutions containing delta-shock waves for nonstrictly hyperbolic conservation laws, Quart. Appl. Math. LV, 361-373 (1997)

[6] D. J. Korchinski, Solution of a Riemann problem for a $2 \times 2$ system of conservation laws possessing no classical weak solution, Ph.D. thesis, Adelphi University, 1977

[7] D. C. Tan, T. Zhang, and Y. X. Zheng, Delta-shock waves as limits of vanishing viscosity for hyperbolic systems of conservation laws, J. Differential Equations 112, 1-32 (1994)

[8] T. Zhang and G. Q. Chen, Some fundamental concepts about system of two spatial dimensional conservation laws, Acta Math. Sci. 6, 463-474 (1986)

[9] J. M. Greenberg and A. Y. Leroux, A well-balanced scheme for the numerical processing of source terms in hyperbolic equations, SIAM J. Numer. Anal. 33, 1-16 (1996)

[10] E. Weinan, Yu. G. Rykov, and Ya. G. Sinai, Generalized variational principles, global weak solutions and behavior with random initial data for systems of conservation laws arising in adhesion particle dynamics, Comm. Math. Phys. 177, 349-380 (1996) 